EMBRYARIDDLE
Aeronautical University

SCHOLARLY COMMONS
International Journal of Aviation, Aeronautics, and Aerospace

\title{
Determination of Aerodynamic Forces and Control Requirement during Ground Effect
}

Ambuj Srivastava

Indian Institute of Technology Kanpur, ambujs@iitk.ac.in

Follow this and additional works at: https://commons.erau.edu/ijaaa

Part of the Other Aerospace Engineering Commons

\section{Scholarly Commons Citation}

Srivastava, A. (2019). Determination of Aerodynamic Forces and Control Requirement during Ground Effect. International Journal of Aviation, Aeronautics, and Aerospace, 6(4). https://doi.org/10.15394/ ijaaa.2019.1413

This Concept Paper is brought to you for free and open access by the Journals at Scholarly Commons. It has been accepted for inclusion in International Journal of Aviation, Aeronautics, and Aerospace by an authorized administrator of Scholarly Commons. For more information, please contact commons@erau.edu. 


\section{Introduction}

The present work outlines the estimation of the effect of ground proximity on typical longitudinal aerodynamic forces, variation in the trim angle of attack, and elevator required for trimming the aircraft. The conventional methodology utilizes equations of motion and flight data for the angle of attack, flight path angle, thrust, velocity, etc. for determining longitudinal aerodynamic force during any flight phase. The current work presents a methodology, which utilizes only flight datafor height above ground for the estimation of longitudinal aerodynamic forces. The effect of ground proximity exclusively influences two safety-critical flight phases,i.e., Take-off and Landing of an aircraft.

The ground effect studies are crucial for accurate representation of take-off and landing and especially the touchdown sink rate. The presence of ground acts as a reflecting surface and consequently augments the pressure beneath the wing,which in turn reduces the sink rate. The primary effects of the ground proximity are to decrease the downwash angle experienced at the horizontal tail, escalation in lift curve slope of the wing, and horizontal tail and a decrement in the induced drag. The behavior of overall drag also depends on the parasite drag experienced by an aircraft during such flight condition (Etkin, 1972).

Weiselsberger elucidated the most nascent theory of ground effect through a mathematical model founded on classical Prandtl's lifting line theory (Prandtl, 1923; Wieselberger, 1922). The critical safety aspect in ground effect studies attracted many types of research towards the development of enhanced understanding. The researches include conceptual ground effect modeling, wind tunnel tests, numerical investigations, and the estimation through flight tests (Campbell, Hassel, Jr., \& Thomas, 1978; Chen \& Schweikhard, 1985; Corda, Stephenson, Mark, Frank, \& Curry, 1994; Coulliette \& Plotkin, 1996; Curry, 1997; Curry \& Owens, 2003; Kemmerly \& Paulson, 1989; Lange \& Moore, 1979; Lee, Lan, \& Muirhead, 1989; Mantle, 2016; Mills, 2017; Nuhait, 1995; Philips, 1985; Chang \& Muirhead, 2012; Qu, Jia, Wang, Liu, \& Agarwal, 2014/2015; Schweikhard, 1967; Staufenbiel, 1978; Staufenbiel \& Schlichting, 1988; Tani, Taima \& Simidu, 1937; Zerihan \& Zhang, 2000). The continuous efforts in pursuit of utilizing advantages of ground effect have lead to the development of an entirely different category of vehicles, commonly known as Wing-In-Ground (WIG) aircraft. The WIG utilizes the concept of ground effect for a faster and economical mode of transportation. The augmented aerodynamic lift and reduced induced drag during ground effect lead to higher cruise speed and low fuel consumption (Amir, Maimun, Mat, \& Saad, 2016; Lange \& Moore, 1979; Leonard, 2001; Tofa, Ahmed, Maimun, Ahmed, \& Jamei, 2014; Wang, Teo, Khoo, \& Goh, 2013).

The present work utilizes flight data of a high wing, twin turboprop towards the prediction of aerodynamic forces in the presence of the ground effect. The intention is to demonstrate that the proposed methodology can also be utilized 
as a viable alternative method for the prediction of aerodynamic forces in ground effect with excellent accuracy and least computational effort. The methodology would be advantageous, especially for flight vehicle with complex flight dynamics. Under such circumstances, the development of fundamental equations of motion which precisely depict the motion would be highly complex and incur computational burden. The longitudinal aerodynamic forces experienced by the aircraft in the presence of ground effect are compared for establishing the efficacy of the methodology.

The original contributions of this paper are:

i) The postulation of a methodology for estimating longitudinal aerod ynamic forces, angle of attack, and elevator angle required for trimming the aircraft during landing in the presence of the ground effect.

ii) The comparative evaluation of longitudinal aerodynamic forces as estimated by employing the proposed methodology and conventional methodology in order to establish the efficacy of the proposed methodology.

The following sections will provide information about the aircraft used for performing flight test maneuver, the maneuver which simulates the ground effect, measurements of flow field variables, the methodologies employed in the current work for estimating longitudinal aerodynamic forces in the presence of ground effect factor,subsequently results obtained by the two methods, comparative analysis and the conclusions inferred from the results.

\section{Flight Data Generation}

The requisite flight data is obtained by conducting constant alpha ground effect maneuver on a high wing, twin turboprop aircraft. The aspect ratio of the wing is $\sim 9.0$. The aircraft is certified as commuter aircraft under medium all up weight category as per FAR 23 guidelines. Figure 1 presents the three view of the aircraft.

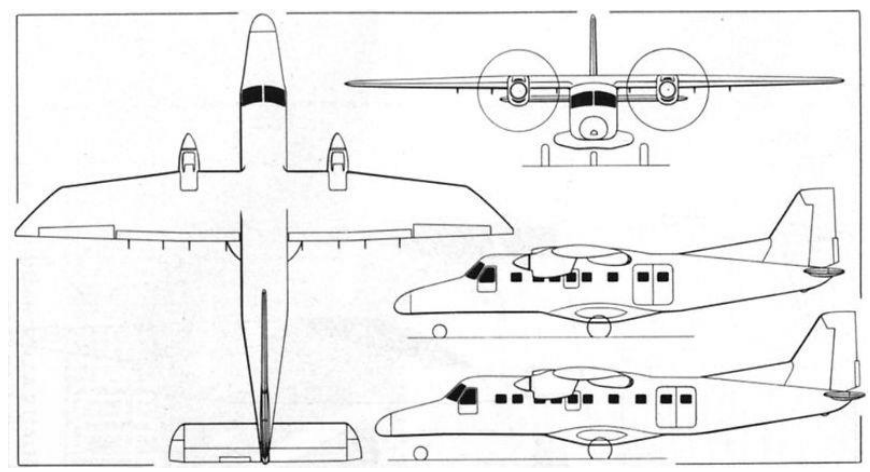

Figure 1. Three view of twin-turboprop aircraft. 
The constant alpha maneuver was appropriately explained by Schweikhard (1967). The Constant -Alpha approach involves aircraft flight at a nearly constant angle of attack and constant power setting during the approach to the runway. When flight vehicle experiences change in the flight path, sink rate, velocity, and control surface position during landing, then it is an indication of the onset of ground effect which ultimately leads to change in longitudinal aerodynamic coefficients, i.e., lift, drag and pitching moment characteristics of aircraft. During the entire maneuver, the aircraft maintains near a constant angle of attack and throttle settings for obviating the occurrence of an error in measurement. Any change in these parameters during the entire maneuver until the touch down will lead to the inappropriate prediction of ground effect and is one of the prime sources of error. The entire constant-alpha maneuver comprises of two zones viz. the first zone is Outside Ground Effect (OGE) which is at the height of more than one wingspan above the ground whereas, the second zone is Inside Ground Effect (IGE) which is for all height below unit wingspan until touch down of aircraft. The initiation point of constant-alpha ground effect maneuver is at a height above screening height, i.e., $\sim 50 \mathrm{ft}$ (Outside Ground Effect zone) and then the aircraft strictly adheres to the primary condition of maintaining constant alpha and throttle settings until the touch down of aircraft. However, due to the practical limitation of maintaining safe \& acceptable limits of horizontal \& vertical speeds, the available landing distance takes the precedence. The range of AOA is maintained to ensure STOL characteristics of aircraft. The range of elevator deflection is also maintained to ensure that the elevator deflection available limit is strictly adhering to forward center of gravity limit of aircraft. All the flight maneuvers ensure that the aircraft weight and center of gravity are as per the limitations of aircraft. The flight path simulates standard landing scenario during a routine flight. Moreover, the throttle settings and the rate at which aircraft is losing height in 'Out of Ground Effect' regime, are also established very carefully such that the aircraft is always in equilibrium glide and not in Phugoid type oscillations.

The aircraft was appropriately instrumented to record flight variables like velocity,linear acceleration, pitch angle, outside air temperature, height above ground, pitch rate, and elevator deflection. The thrust magnitudes are calculated during post-flight analysis. The weight is calculated by utilizing onboard fuel flow counters. The angle of attack is a derived parameter by using the flight path angle and pitch angle. The time history of flow-field variables during the entire maneuver. Figure 2 presents the time history plot of flow field variables. The height above ground and TAS shows a decreasing trend with the progress of time for landing, the flight path angle has shown some scatter at all instants of outside ground effect, but at all instances within ground effect, flight path angle progressively decreases during landing. The elevator deflection required during the entire landing flight phase maintains a trimmed flight. The AOA and flight path angle have shown a 
near uniform behavior during the entire constant-alpha maneuver. The crosswinds will have an impact on data accuracy during the ground effect. Therefore a limit of cross winds less than 5 knots is adopted for improved accuracy.
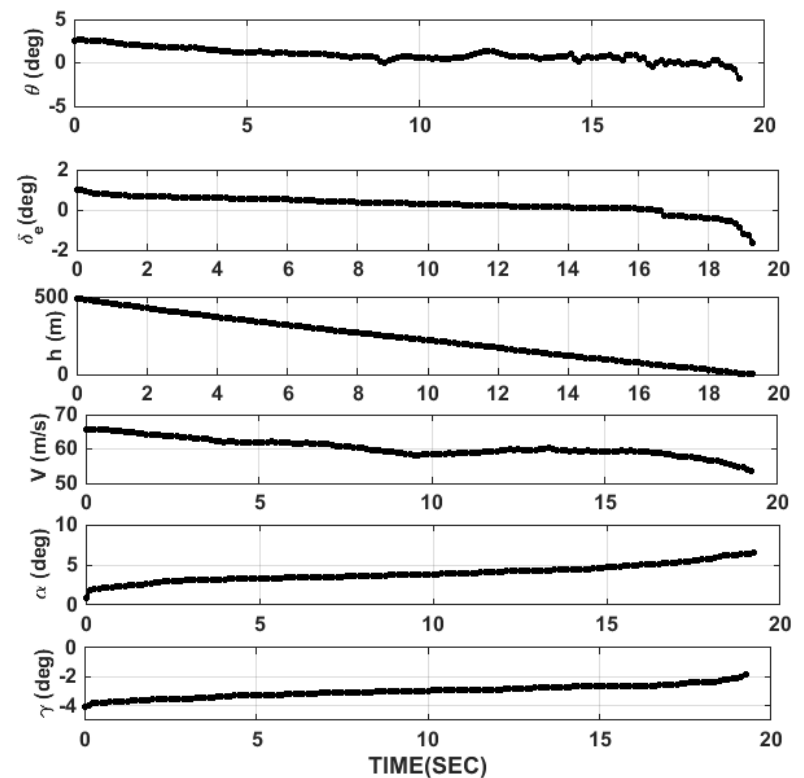

Figure 2. Time history plot of flow field variables during landing.

\section{Proposed Methodology}

\section{Methodology}

The proposed methodology adopts the notion of varying ground effect with the height above ground.The aircraft experiences ground effect at nearly one wing span but negligible 1.4 , which increases to $\sim 9 \%$ at half wing span and becomes $\sim 47 \%$ at one-tenth of wing span (Hurt, 1965). The methodology estimates the residual percentage level of downwash at all values of dimensionless height above ground,i.e., h/b in linearly decreasing steps, assuming full downwash at unit wingspan. The complete flight data of height above ground is divided into an equal number of steps and also adhering to the aforementioned downwash levels. The intention of the current work is to demonstrate that the linearly decreasing approximation of residual downwash level is capable of predicting the aerodynamic lift and drag in the presence of the ground effect. Moreover, the estimated values of aerodynamic lift and drag will also match well with the estimated values from the conventional method.

The linearly decremented value of the residual percentage level of downwash is subsequently utilized to estimate effective downwash angle through equation 1 . 


$$
\varepsilon=\varepsilon_{0}+\left(\frac{d \varepsilon}{d \alpha}\right) \alpha_{t r i m}
$$

Where,

$$
\begin{aligned}
& \varepsilon_{0}=\text { Downwash angle at zero angle of attack } \\
& \frac{d \varepsilon}{d \alpha}=\text { Downwash distribution during landing } \\
& \alpha_{\text {trim }}=\text { Trimmed value of angle of attack }
\end{aligned}
$$

The value of the effective angle of attack, which delivers the difference between the geometric angle of attack and the downwash angle can be obtained by using equation 2 . The pilot maintains a nearly constant angle of attack during the entire maneuver.

$$
\alpha_{\text {eff }}=\alpha-\varepsilon
$$

The effective angle of attack progressively increases as downwash continue to deplete with the decrease in height above the ground. The methodology involves the estimation of the additional angle of attack on the top of the effective angle of attack at the height of unit wingspan. This additional effective angle of attack progressively increases with the increasing proximity to the ground. This additional effective angle of attack results in additional lift coefficient, which is entirely due to ground proximity, i.e., ground effect. As deliberated earlier that the trim angle of attack continuously varies with the continuous decrease in height above ground within unit wingspan, but during the entire ground effect maneuver, pilot maintained a constant value of angle of attack. The pilot experiences a continuous variation in elevator deflection required for trimming the aircraft while maintaining a constant value of angle of attack. This aspect of continuous variation in elevator deflection required to trim the aircraft is thus an indicat or of ground effect.

The longitudinal aerodynamic forces,i.e., lift and drag during the entire maneuver are estimated by using equations 3 and 4, respectively.

$$
\begin{aligned}
& C_{L}=C_{L 0}+\frac{d C_{L}}{d \alpha} \alpha_{e f f} \\
& C_{D}=C_{D 0}+K C_{L}^{2}
\end{aligned}
$$

The value of aerodynamic lift and drag in Outside Ground Effect (OGE) and Inside Ground Effect (IGE) regimes are estimated separately by using equations $3 \& 4$ only. The inside ground effect values of lift and drag are denoted as $C_{L(I G E)}$ and $C_{D(I G E)}$ respectively whereas $C_{L(O G E)}$ and $C_{D(O G E)}$ for outside ground effect regime. It is imperative to mention that during the flight of aircraft in ground proximity, the required elevator deflction for trimming the aircraft continuously varies. The elevator deflection required for trimming the aircraft for all heights within unit wing span is estimated by using equation 5 . The primary 
intention of estimating elevator deflection required for trimming is to demonstrate its variation with increasing proximity to ground.

$$
C_{m}=C_{m 0}+C_{m \alpha} \alpha_{t r i m}+C_{m \delta e} \delta_{e t r i m}
$$

The present section explained the notion of deriving the proposed methodology, procedure to determine residual downwash at every incremental step within unit wingspan, procedure to obtain effective downwash angle and effective angle of attack, process to estimate longitudinal aerodynamic lift and drag in the presence of ground effect and the elevator angle required for trimming the aircraft at each incremental level.

\section{Conventional Method}

The conventional method for estimating the longitudinal aerodynamic lift and drag in ground proximity involves a number of recorded flight variables such as linear acceleration, velocity, thrust magnitude, the instantaneous mass of the aircraft through fuel flow counters, pitch angle, height above ground, outside air temperature, etc. The angle of attack is a derived parameter by using the flight path angle and pitch angle. Equations 6-9 present the estimation of longitudinal aerod ynamic lift and drag.

$$
\begin{aligned}
& C z=\frac{m a_{Z}}{\bar{q} S_{r e f}} \\
& C x=\frac{\left(m a_{X}-T\right)}{\bar{q} S_{r e f}} \\
& C_{L}=-C z \cos \alpha+C x \sin \alpha \\
& C_{D}=-C x \cos \alpha-C z \sin \alpha
\end{aligned}
$$

The aerodynamic lift and drag for the entire ground effect maneuver, which is inclusive of both outside ground effect and inside ground effect regime are estimated by the conventional method. A similar methodology was used by Curry and Owens (2003) for the estimation of longitudinal aerodynamic forces in the ground proximity. The crucial observation was that the aerodynamic lift followed a power law formulation with the varying dimensionless height above ground, and the aerodynamic drag exhibited an oscillating behavior.

An assessment efficacy of the proposed methodology is done by carrying out a comparative analysis of the behavior exhibited by the estimated longitudinal aerodynamic forces by both the above-mentioned methods. 


\section{Results and Discussion}

The complete analysis includes the determination of the variation of percentage residual downwash, effective angle of attack, aerod ynamic lift, and drag and the required elevator deflection for trimming the aircraft with the decreasing non-dimensional height above ground $(\mathrm{h} / \mathrm{b})$ within unit wingspan. A comparative analysis of the estimated aerodynamic lift and drag in ground proximity from both the methods is additionally provided for assessing the efficacy of the proposed methodology.

\section{Variation of Percentage Residual Downwash}

As deliberated earlier that the decremented percentage residual downwash is estimated by assuming the linear decrement. Figure 3 shows the variation of residual percentage downwash level with decreasing non-dimensional height above ground. The figure indicates that residual downwash level is reduced to only $9 \%$ until height above the ground is around half the wingspan, which further reduces to $47 \%$ when height above the ground is approximately one-tenth of wingspan.

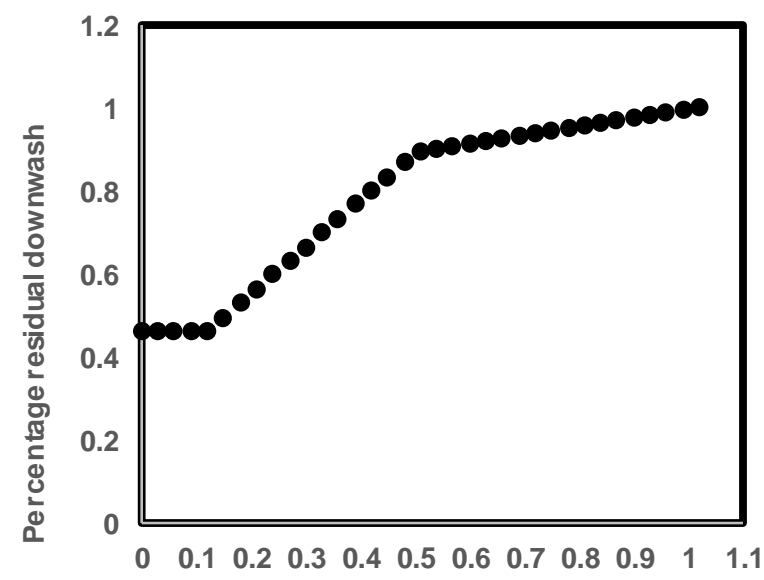

Non-dimensional height above ground (h/b)

Figure 3. Variation of percentage residual downwash with non-dimensional height above ground.

\section{Variation of Effective Angle of Attack}

As mentioned earlier, that the effective downwash angle is estimated by using equation 1 and the percentage residual downwash. The effective downwash angle reduces from $1.973 \mathrm{deg}$ at the height of unit wingspan to $0.909 \mathrm{deg}$ at the height of around one-tenth of wingspan. The apparent effect of a decrease in downwash angle is the change in the effective angle of attack, which is estimated by using equation 2 . The effective angle of attack exhibits a variation of 1.0 degree 
at the height above ground around unit wingspan to around 2.06 degree at the height above ground of around one-tenth of the wingspan. Figure 4 shows the variation of effective downwash angle with non-dimensional height above ground $(\mathrm{h} / \mathrm{b})$. The effective downwash angle exhibits a decreasing trend with the decreasing

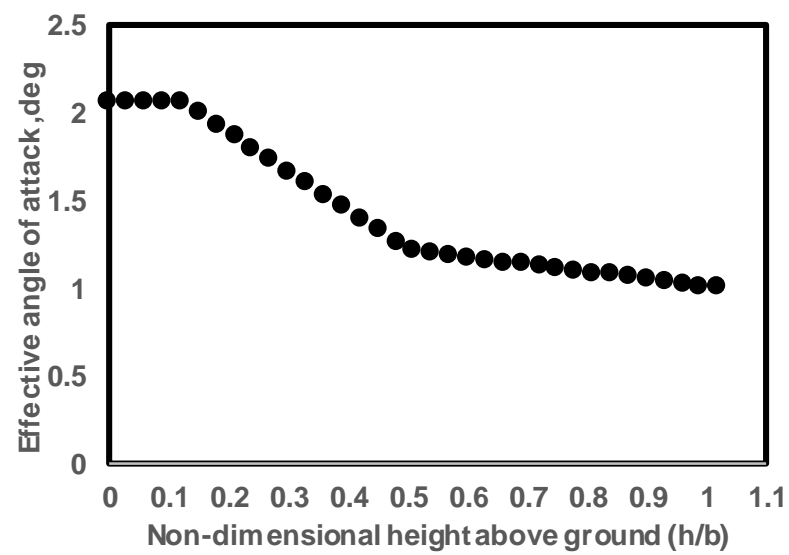

Figure 4. Variation of effective angle of attack with non-dimensional height above ground.

\section{Variation of Aerodynamic Lift and Drag}

The effect of ground proximity on longitudinal aerodynamic forces, i.e., lift and drag from the proposed methodology is obtained by using equations 3 and 4, respectively. The variation of aerodynamic lift and drag with the height above ground within until wingspan is shown in Figures 5 and 6, respectively. To deliver an improved appreciation of the lift and drag variation inside the ground effect regime, the lift and drag coefficient inside ground effect are normalized with respect to their magnitudes outside ground effect. The normalized lift coefficient exhibits a continuous decrease from $\sim 1.255$ at the non-dimensional height above ground close to 0.1 , whereas it decreases to a nearly constant value of 1.0 at the nondimensional height above ground equal to 1.0 and above. The above behavior agrees to the academic understanding of the ground, i.e., the ground effect vanishes at the height above ground equal to unit wingspan. The variation of aerodynamic lift coefficient follows a near power law variation with the decreasing height above ground. The aerodynamic drag inside ground effect exhibits that aerodynamic drag coefficient shows oscillatory and insignificant variation with the height above ground up to almost half the unit wingspan, and subsequently exhibits a sharp decline until the nondimensional height above ground is equal to 0.1 . Curry and Owens (2003) have demonstrated that aerodynamic lift inside ground effect follows a progressive increment as per power law and aerodynamic drag oscillates without any specific trend. 


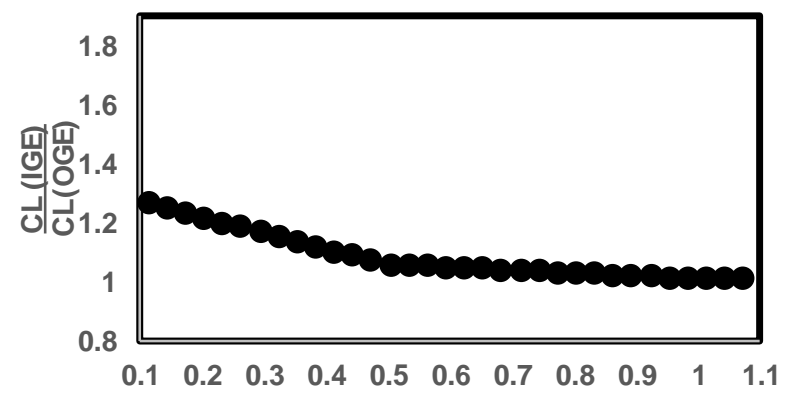

Non dimensional height above ground (h/b)

Figure 5. Variation of normalized lift coefficient with non-dimensional height above ground.

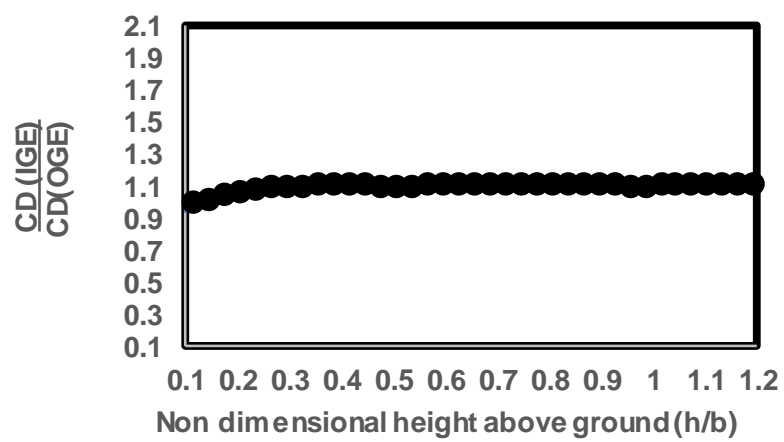

Figure 6. Variation of normalized drag coefficient with non-dimensional height above ground.

\section{Variation Elevator Required for Trimming the Aircraft}

The variation in elevator required for trimming between outside ground effect regime and inside ground effect regime is an excellent indicat or of the onset of the ground effect and its magnitude. Figure 7 displays the variation of elevator required for trimming the aircraft with the non-dimensional height above ground. The change in elevator required for trimming the aircraft increases from $-0.64 \mathrm{deg}$ to $-0.86 \mathrm{deg}$. The increase in the required elevator deflection for trimming the aircraft gives a feel of ground proximity to the pilot. 


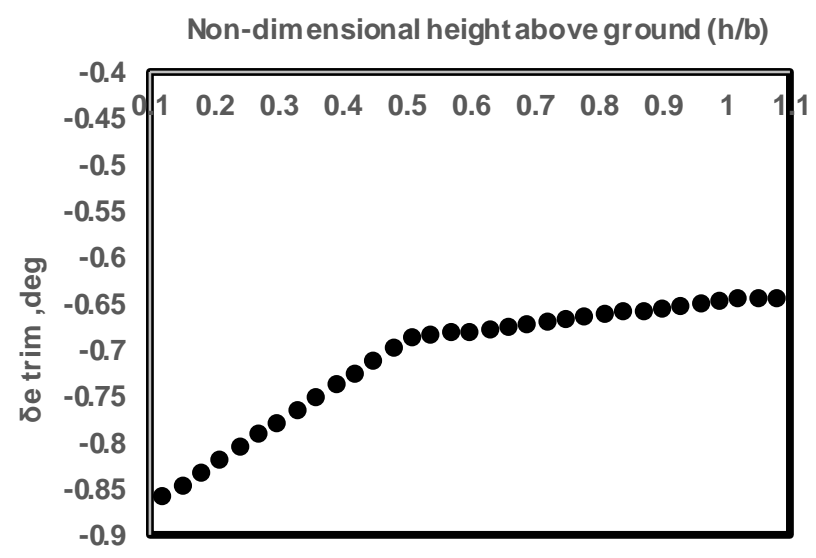

Figure 7. Variation of elevator required for trimming the aircraft with nondimensional height above ground.

\section{Comparative Evaluation of Aerodynamic Forces Estimated by Proposed and Conventional Method}

A comparative evaluation of the aerodynamic forces as estimated by the two methods is also made in current work for assessing the efficacy of the proposed methodology. The aerodynamic lift and drag in the presence of ground effect by the conventional method are estimated by using equations 6-9 (Curry \& Owens, 2003). Figure 8 depicts the comparison of aerodynamic lift estimated by the two methods inside ground proximity regime. The normalized lift coefficient estimated by the proposed methodology varies from $\sim 1.255$ at the non-dimensional height above ground around 0.1 to $\sim 1.0$ at the height around unit wingspan. The normalized lift coefficient estimated by the conventional method varies from $\sim 1.78$ at the height above ground around one-tenth of wingspan to $\sim 1.0$ at the height of unit wing span above ground. The comparative evaluation indicates that both the methods estimate a gradual rise in the augmentation of aerodynamic lift for all the values of height above ground less than half of the wingspan. The probable reason for the prediction of the different magnitude of aerodynamic lift by the two methods could be the assumption made during the formulation of the equation and the presence of inevitable noise in the measured flight data. Figure 9 depicts the comparison of aerodynamic drag estimated by the two methods inside ground proximity regime. The comparative evaluation signifies that the proposed methodology hints the insignificant variation in the aerodynamic drag for all values of height above ground less than the unit wingspan whereas, the conventional method indicates small and oscillatory nature of aerodynamic drag inside ground effect (Curry \& Owens, 2003). The probable reason for the oscillatory behavior is the smaller magnitude of force variation along drag axis as compared to more 
significant force along the lift axis. The presence of the measurement noise of the same order as that of the drag variation can lead to oscillatory behavior of aerodynamic drag. The common observation by the two methods is the small magnitude of aerodynamic drag inside the ground effect regime.

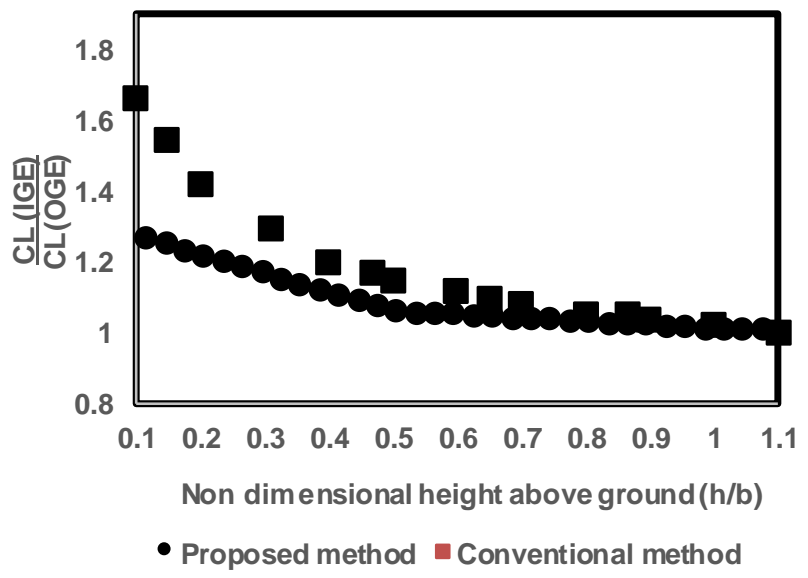

Figure 8. Variation of normalized aerodynamic lift with non-dimensional height above ground.

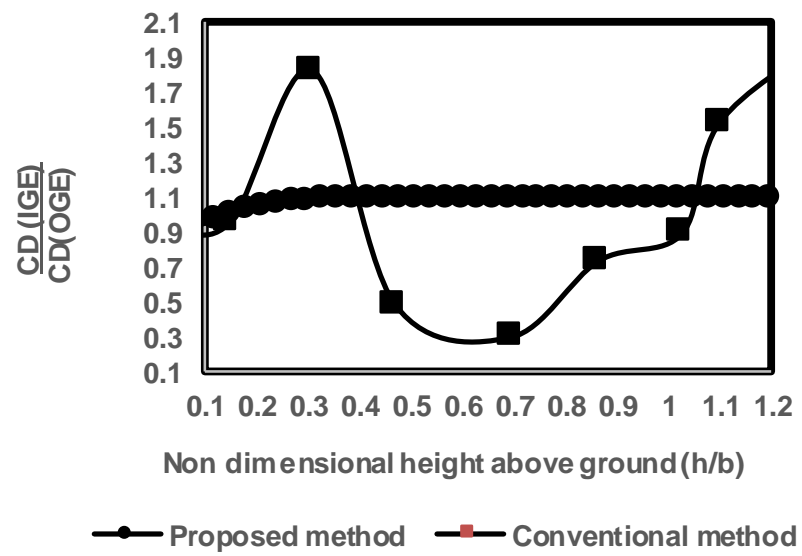

Figure 9. Variation of normalized aerodynamic drag with non-dimensional height above ground.

\section{Conclusions}

The sections mentioned above outlined the ground effect phenomenon, the application of the proposed methodology for estimating downwash angle, effective angle of attack, a variation of aerodynamic forces and elevator required for trimming the aircraft inside ground effect. The proposed methodology is successful 
in predicting the trend of these variables with reasonable accuracy. The proposed methodology would be especially beneficial in the circumstances where, a limited amount of flight data is available, the scope of measuring flight data is a constraint, and complex system dynamics of flight vehicle. The development of a precise mathematical model for such flight vehicles is very challenging, and the solution of equations becomes very tedious accompanying with additional computation effort. Under such circumstances, the proposed methodology can be utilized as a viable alternative method for estimating longitudinal aerodynamic forces inside ground effect regime.

The future scope of the current work is to predict the variation of aerodynamic forces inside ground effect when the ground effect maneuver is performed with varying sink rates. Another scope is to use the methodology for predicting the aerodynamic forces for smaller values of non-dimensional height above ground, where the non-linearity predominates the scenario. The work can also be utilized for the estimation and refining the aerodynamic forces acting on Wing-in-ground (WIG) aircraft towards optimizing the fuel consumption and the handling qualities of the aircraft. 


\section{References}

Amir, M. A. U., Maimun, A., Mat, S., \& Saad, M. R. (2016). Computational analysis of aerodynamic characteristics for wing-in-ground effect craft in lateral stability. IOP Conf. Series: Materials Science and Engineering. doi:10.1088/1757-899

Campbell, J. P., Hassel, J., L., Jr., \& Thomas, J. L. (1978). Ground effects on lift for turbofan powered-lift STOL aircraft. Journal of Aircraft, 15(2), 78-84. doi:10.2514/3.58318

Chang, R. C., \& Muirhead, U. V. (2012). Effect of sink rate on ground effect of low aspect ratio wings. Journal of Aircraft, 24(3), 176-180. doi:10.2514/3.45413

Chen, Y., S., \& Schweikhard, W. G. (1985). Dynamic ground effects on a two dimensional flat plate. Journal of Aircraft, 22(7), 638-640. doi:10.2514/3.45179

Corda, S., Stephenson T., Mark, B., Frank, W., \& Curry, E. R. (1994, September). Dynamic ground effects light test of an F-15 aircraft. NASA TM-4604.

Coulliette, C., \& Plotkin, A. (1996). Aerofoil ground effect revisited. The Aeronautical Journal, 100(92), 65-74. doi:10.1017/S0001924000027305

Curry, E. R. (1997, August). Dynamic ground effect for a cranked arrow wing airplane. NASA TM 4799.

Curry, E. R., \& Owens, R. L. (2003). Report on ground-effect characteristics of the Tu144 supersonic transport airplane. NASA/TM-2003-212035.

Etkin, B. (1972). Dynamics of atmospheric flight. New York, NY: John Wiley \& Sons.

Hurt, H., H. (1965). Aerodynamics for naval aviators. Retreived from https://www.faa.gov/regulations_policies/handbooks_manuals/aviation/media/00 80t-80.pdf

Kemmerly, G. T., \& Paulson, J. W. (1989). Investigation of a moving-model technique for measuring ground effects. NASA TM-4080.

Lange, R., H., \& Moore, J., W. (1979). Large wing-in-ground effect transport aircraft. Journal of Aircraft, 17(4), 206-266. doi:10.2514/3.57898

Lange, R., H., \& Moore, J., W. (1979). Large wing-in-ground effect transport aircraft. Journal of Aircraft, 17(4), 206-266. doi:10.2514/3.57898

Lee, P.-H., Lan, C., E., \& Muirhead, U. V. (1989). Experimental investigation of dynamic ground effect. Journal of Aircraft, 26(6), 497-512. doi:10.2514/3.45793

Leonard, N. J. (2001). Wing in ground effect aircraft: An airlifter of the future. WrightPatterson Air Force Base, OH: Air Force Institute of Technology. Retreived from https://apps.dtic.mil/dtic/tr/fulltext/u2/a430859.pdf

Mantle, P. J. (2016). Induced drag of wings in ground effect. The Aeronautical Journal, 120(1234), 1867-1890. doi:10.1017/aer.2016.106

Mills, N. L. (2017). Calculation of air displaced by a wing. Journal of Aeronautics and Aerospace Engineering, 6(4), 1000204.

Nuhait, A. O. (1995). Unsteady ground effects on aerodynamic coefficients of finite wings with camber. Journal of Aircraft, 32(1), 186-192. doi:10.2514/3.46699 
Philips, D., J., (1985, January). Downwash in the plane of symmetry of an elliptically loaded wing. NASA-TP-2414.

Prandtl, L. (1923). Applications of modern hydrodynamics to aeronautics. Retreived from https://ntrs.nasa.gov/search.jsp?R=19930091180

Qu, Q., Jia, X., Wang, W., Liu, P., \& Agarwal, R. K. (2014). Numerical simulation of the flowfield of an airfoil in dynamic ground effect. AIAA Journal, 51(5), 1659-1662. doi:10.2514/1.C032452

Qu,Q., Jia, X., Wang, W., Liu, P., \& Agarwal, R. K. (2015). Airfoil aerodynamics in ground effect for wide range of angles of attack. AIAA Journal, 53(4), 1-14. doi:10.2514/1.J053366

Schweikhard, W. G. (1967). A method for in-flight measurement of ground effect on fixed-wing aircraft. Journal of Aircraft, 4(2), 101-104. doi:10.2514/3.43804

Staufenbiel, R. (1978). Some nonlinear effects in stability and control of airplane of wing-in-ground effect vehicles. Journal of Aircraft, 15(8), 541-544. doi:10.2514/3.58404

Staufenbiel, R. W., \& Schlichting, U.-J. (1988). Stability of airplane in ground effect. Journal of Aircraft, 25(4), 289-294. doi:10.2514/3.45562

Tani, I., Taima, M., \& Simidu, S. (1937, September). The effects of ground on the aerodynamic characteristics of a monoplane wing. Tokyo Imperial University, Japan, Aero Research Institute Report 156.

Tofa, M. M., Ahmed, Y. M., Maimun, A., Ahmed, Y. M., \& Jamei, S. (2014). Experimental investigation of a wing-in-ground effect craft. The Scientific World Journal, Paper ID 489308. doi:10.1155/2014/489308

Wang, H., Teo, C. J., Khoo, B. C., \& Goh, C. J. (2013). Computational aerodynamics and flight stability of wing-in-ground (WIG) craft. 7th Asian-Pacific Conference on Aerospace Technology and Science. doi:10.1016/j.proeng.2013.12.002

Wieselberger, C. (1922). Wing resistance near the ground. NACA TM-77.

Zerihan, J., \& Zhang, X. (2000). Aerodynamics of a single element wing in ground wffect. Journal of Aircraft, 37(6), 1058-1064 .doi: 10.2514/2.2711 


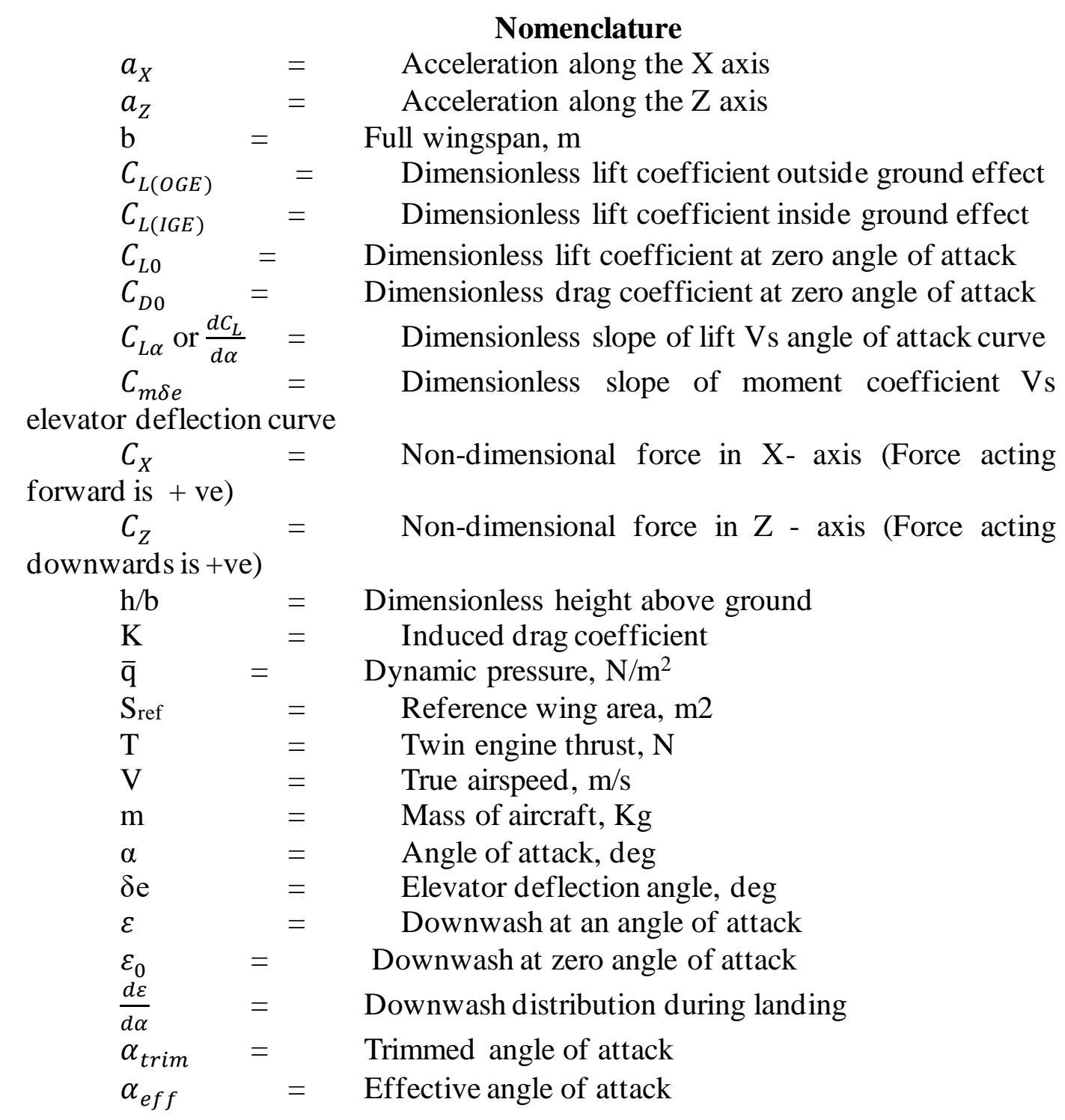

